\title{
Finite Element Treatment of Vortex States in 3D Mesoscopic Cylindrical Superconductors in a Tilted Magnetic Field
}

\author{
Lin Peng ${ }^{a, *}$, Yahua Hu ${ }^{b}$, Zhijian $\mathrm{Li}^{a}, \mathrm{Ke} \mathrm{DenG}^{a}$, Yanyan Zhu ${ }^{a}$, Li Xu ${ }^{a}$ And Yun Zhou ${ }^{c}$ \\ ${ }^{a}$ Department of Physics, Shanghai University of Electric Power, Shanghai 201300, China \\ ${ }^{b}$ College of Nanhu, Jiaxing University, Jiaxing, Zhejiang 314001, PR China \\ ${ }^{c}$ Department of Physics, China Jiliang University, Hangzhou 310018, China
}

(Received July 3, 2017; in final form October 21, 2017)

\begin{abstract}
The time-dependent Ginzburg-Landau equations have been solved numerically by a finite element analysis for the mesoscopic superconducting samples with cylindrical shape in a uniform axial magnetic field. We obtain the different vortex patterns as a function of the applied field perpendicular to its surface. We find that multi-vortex states are ground state in three-dimensional mesoscopic cylinders. These results show that our approach is an effective and useful to interpret experimental data on vortex states in the mesoscopic superconductors.
\end{abstract}

DOI: 10.12693/APhysPolA.133.152

PACS/topics: three-dimensional mesoscopic cylinders, TDGL equations, finite element method, vortex states

\section{Introduction}

Modern lithography techniques enable one to create mesoscopic superconducting structures of varied geometries $[1,2]$. Due to the interaction between vortices and sample boundaries, vortex configurations strongly dependent on the size and geometry of mesoscopic samples whose dimensions are of the order of the penetration depth $\lambda$ or the coherence length $\xi$. For example, strong confinement leads to the formation of the giant vortex state [3-7] and multivortex state [8-14], which are energetically less favorable in bulk type-II superconductors [15]. The vortex-antivortex states are easily stabilized in an inhomogeneous magnetic field [16]. Recent theoretical studies have shown that vortex-antivortex states can also be stabilized in submicron superconductors even in a homogeneous magnetic field [17]. Vortices show very rich static and dynamic behavior in the presence of a weak link $[9,12,18]$. A weak link is commonly achieved by two superconducting layers separated by a normal metallic layer, or a weak superconducting region. Vortices in the weak link are found to be more mobile than the ones located in the strong superconducting regions between the weak links leading to distinct dissipation in the system. In experiment, the measured superconducting phase boundary for the mesoscopic $\mathrm{Al}$ square shows the direct experimental evidence for these symmetry-induced vortex-antivortex pairs [19].

An experimental investigation was made of flux jumps and irreversible magnetization of mesoscopic $\mathrm{Al}$ superconducting rings, which indicated that the change of vorticity with magnetic field could be larger than unity [20]. A direct observation of vortex states in small superconducting disks for vorticity $L=0$ to 40 was also reported [21].

*corresponding author; e-mail: plpeng@shiep.edu.cn
In previous theoretical studies of vortex states, most of them were performed using the Ginzburg-Landau (GL) theory but in the two-dimensional limit. The numerical investigations of the three-dimensional (3D) GL formalism were also performed using a self-consistent approach and the finite difference technique for solving two coupled GL equations $[3,10,11,18]$. Since recently, such superconducting samples (cylindrical wires and tubes) were experimentally realized [22]. In this paper, we investigate the 3D time-dependent Ginzburg-Landau (TDGL) equations in the order parameter $\psi$ of the Cooper-pair condensate for fully 3D cylindrical samples in the basis of finite-element method (FEM) [12, 23], which is proved to be an effective method for the mesoscopic superconductors with a complex geometry [23].

The paper is organized as follows. In Sect. 2, we show the derived TDGL equations and explain the numerical method and procedure we use in the calculations. In Sect. 3, we analyze the results obtained for the samples with cylindrical symmetry. Our results are finally summarized in Sect. 4.

\section{Time-dependent Ginzburg-Landau model}

We consider the mesoscopic superconducting hollow cylinders with inner radius $R_{\mathrm{i}}$, outer radius $R_{o}$ and the height $d$. Figure 1 shows the schematic view of the cylinder under an arbitrarily tilted field, where the rotation angle $\theta$ is measured with respect to the major $(z)$ axis: $H=H_{0} \cos \theta$. The plane of rotation is referred to as the $y$ - $z$ plane, and we introduce the rotation for fields oriented parallel and perpendicular to the major axis: $H_{\|} \equiv H\left(\theta=0^{0}\right)$ and $H_{\perp} \equiv H\left(\theta=90^{\circ}\right)$, respectively. The GL theory describes the superconducting state through a complex order parameter $\psi$ for which $|\psi|^{2}$ represents the density of the Cooper pairs. The order parameter and the local magnetic field can be determined by their TDGL equations, which are expressed by

$$
\left(\frac{\partial}{\partial t}+\mathrm{i} \Phi\right) \psi=(\mathrm{i} \nabla+\boldsymbol{A})^{2} \psi+\left(1-|\psi|^{2}\right) \psi
$$




$$
\sigma\left(\frac{\partial \boldsymbol{A}}{\partial t}+\nabla \Phi\right)=\boldsymbol{J}_{s}-\kappa^{2} \nabla \times \nabla \times \boldsymbol{A},
$$

with boundary conditions

$$
\left.\boldsymbol{n} \cdot(-\mathrm{i} \nabla-\boldsymbol{A}) \psi\right|_{\perp, \text { boundary }}=0,
$$

where $\Phi$ is the electric potential, $\boldsymbol{A}$ is the vector potential, $\sigma$ is the electric conductivity, $\boldsymbol{n}$ is the normal unit vector on the surface. The density of the superconducting current $\boldsymbol{J}_{s}$ is given by

$$
\boldsymbol{J}_{s}=\frac{\mathrm{i}}{2}\left(\psi \nabla \psi^{*}-\psi^{*} \nabla \psi\right)-|\psi|^{2} \boldsymbol{A}
$$

We scale the length in units of $\xi=\hbar / \sqrt{2 m\left|\alpha_{0}\right|}$, the order parameter $\psi$ in units of $\psi_{0}=\sqrt{-\alpha_{0} / \beta}$ (with $\alpha_{0}$ and $\beta$ being the GL coefficients [24]), the vector potential $\boldsymbol{A}$ in units of $A_{0}=\sqrt{2} \kappa H_{c} \xi$, the time $t$ in unit of $t_{0}=\pi \hbar / 8 k_{\mathrm{B}} T_{c}$, and the local magnetic field $\boldsymbol{B}=\nabla \times \boldsymbol{A}$ in units of $H_{c 2}=\sqrt{2} \kappa H_{c}$, where $H_{c}$ is the thermodynamical critical field, and $\kappa=\lambda / \xi$ is the GL parameter. Notice that the TDGL equations are gauge invariant under the transformations $\psi^{\prime}=\psi \mathrm{e}^{\mathrm{i} \chi}, \boldsymbol{A}^{\prime}=\boldsymbol{A}+\nabla \chi$, $\Phi^{\prime}=\Phi-\partial \chi / \partial t$. Therefore, we choose the zero-scalar potential gauge, that is, $\Phi=0$ at all times and positions. The free energy of the superconducting state, measured in $F_{0}=H_{c}^{2} V / 8 \pi$ units, is expressed as

$$
\begin{aligned}
F & =\frac{2}{V} \int\left[-|\psi|^{2}+\frac{1}{2}|\psi|^{4}+|(-\mathrm{i} \nabla-\boldsymbol{A}) \psi|^{2}\right. \\
& \left.+\kappa^{2}(\boldsymbol{B}-\boldsymbol{H})^{2}\right] \mathrm{d} V .
\end{aligned}
$$

In principle, the TDGL equations can provide all transient states for a fixed external applied magnetic field. A recent review [25] warns us of the fact that for gapped superconductors TDGL is not strictly valid even above $T_{c}$, while below $T_{c}$ it becomes totally wrong; nevertheless TDGL remains popular because of its simplicity and its ability to reproduce observed phenomena. Kramer and Watts-Tobin [26] generalized TDGL equation so that it should be applicable to gapped superconductors as long as there is local equilibrium, while still retaining some of the simplifying features of the TDGL formalism. The simulations of the vortex states are conducted in (i) field sweep up: we started from the $|\psi|=1$ and slowly increased the magnetic field, after reaching the stationary state, (ii) field sweep down: we started simulation with $|\psi|=0$ and $H>H_{c}$ and decreased the field with small steps. The dimensionless magnetization, which is a direct measure of the expelled magnetic field from the sample, is defined as $M=(\langle B\rangle-H) / 4 \pi$, where $\langle B\rangle$ is the magnetic induction averaged over the mesoscopic superconducting cylinder volume $V$, i.e., $\langle B\rangle=(1 / V) \int B(\boldsymbol{r}) \mathrm{d} V$. Our simulations have been carried out by using $\sigma=1$ and $\kappa=10$ for the system. The normalized time $t / t_{0}=0$ to $t / t_{0}=10^{5}$ for the evolution of the dynamics.

\section{Results and discussions}

We first consider a mesoscopic superconducting cylinder of size $R=5 \xi$ and $d=10 \xi$, as illustrated in Fig. 1 . Figure 2 shows absolute value of the order parameter and corresponding isosurface plots of vortex tubes for three selected vortex configurations at the field $H / H_{c 2}=0.4$, $H / H_{c 2}=0.5$, and $H / H_{c 2}=0.6$. The cylinder is in an external uniform magnetic field $H_{\|}\left(\theta=0^{\circ}\right)$. Three selected vortex configurations display vorticity $L=3,4$ and 5 vortex states, respectively. These three vortex configurations illustrate general features of the vortex tubes inside the cylinder. A vortex tube must reach the surface perpendicularly in order to avoid a supercurrent component pointing outwards the surface.
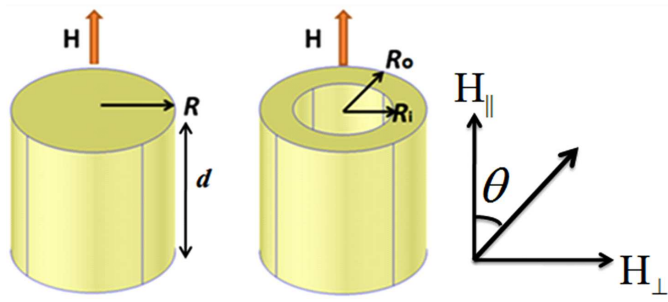

Fig. 1. Considered superconducting geometries: (a) solid cylinders and (b) hollow cylinders. Inner radius (if any) is denoted by $R_{\mathrm{i}}$ and outer one by $R_{o}$.
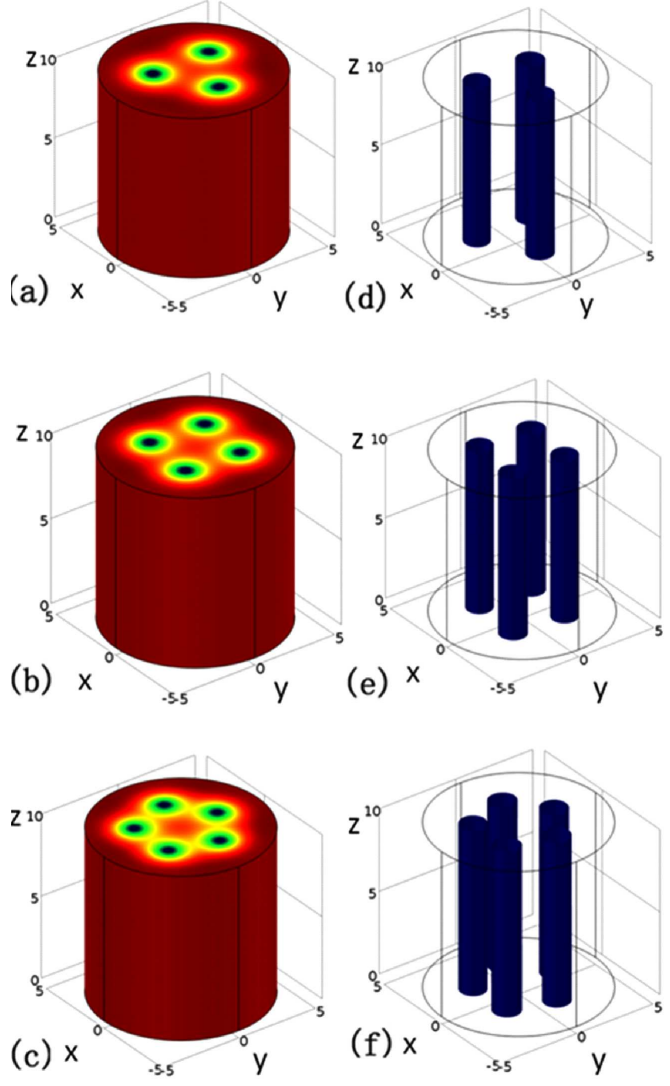

Fig. 2. Absolute value of the order parameter in a 3D mesoscopic superconducting cylinder with the radius $R=5 \xi$ and the height $d=10 \xi$ at the field $H / H_{c 2}=0.4$ (a), $H / H_{c 2}=0.5(\mathrm{~b})$, and $H / H_{c 2}=0.6$ (c). The cylinder is in an external uniform magnetic field $H_{\|}\left(\theta=0^{\circ}\right)$. Parts (d)-(f) show the corresponding isosurface plots of vortex tubes of $(\mathrm{a})-(\mathrm{c})$. Blue to red means the absolute value of the order parameter ranges from minimum to maximum. 

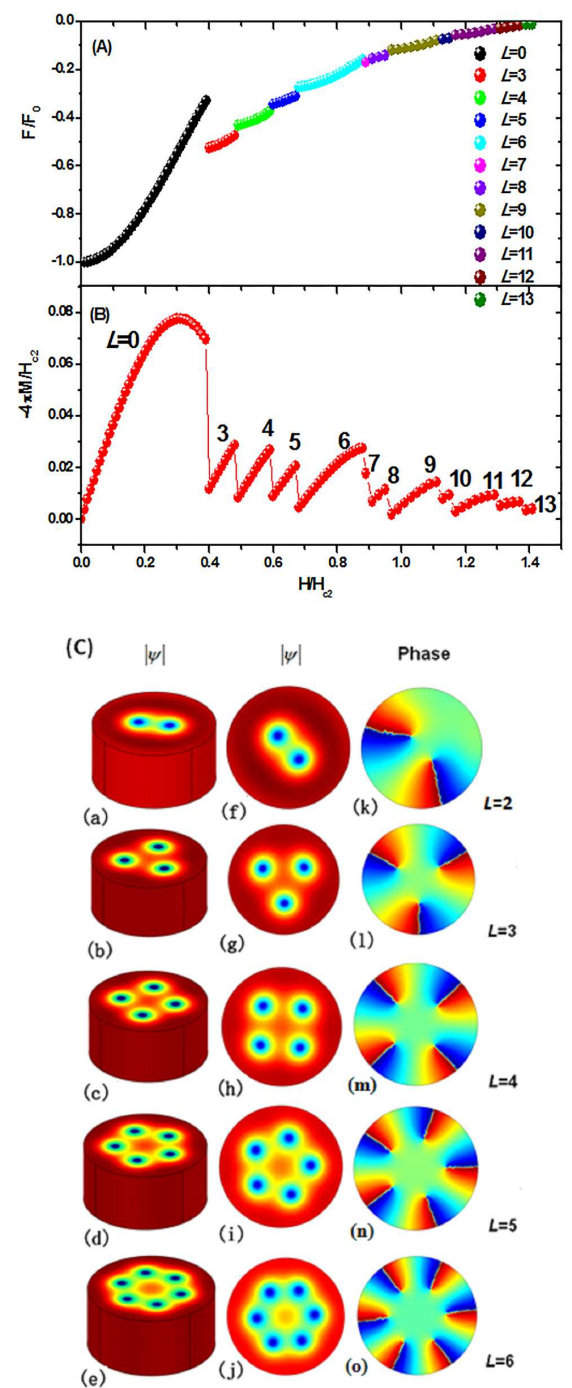

Fig. 3. Free-energy curve $[\mathrm{A}]$, magnetization $[\mathrm{B}]$ and selected $3 \mathrm{D}$ vortex states and corresponding phases $[\mathrm{C}]$ in a $3 \mathrm{D}$ mesoscopic superconducting cylinder with the radius $R=5 \xi$ and the height $d=5 \xi$. The cylinder is in an external uniform magnetic field $H_{\|}\left(\theta=0^{\circ}\right)$. Blue to red means order parameter range from minimum to maximum, whereas for order parameter phase, it indicates $0-2 \pi$.

Figure 3 shows the full free-energy spectrum, magnetization and the corresponding vortex states as a function of applied magnetic field for the cylinder of size $R=5 \xi$ and $d=5 \xi$. The cylinder is in an external uniform magnetic field $H_{\|}\left(\theta=0^{\circ}\right)$. Figure 3 a shows the free energy as a function of $H$ for the cylinder. The lowest energy curve corresponds to the thermodynamic stable pattern, the Meissner phase with no vortices, but only up to the first penetration field $H_{p}=0.39 H_{c 2}$. As the applied field is further raised, three vortex tubes enter the sample. Each jump in the magnetization curve corresponds to a transition between different vortex states (Fig. 3b). We find the multi-vortex states with vorticity $L=2,3,4$, $5,6,7$ and 8 for $0.39 H_{c 2}<H<0.95 H_{c 2}$, and the gi-

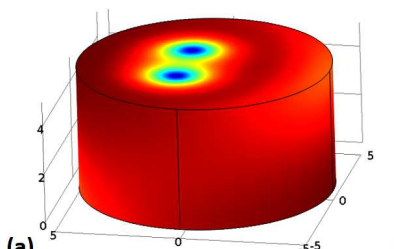

(a)

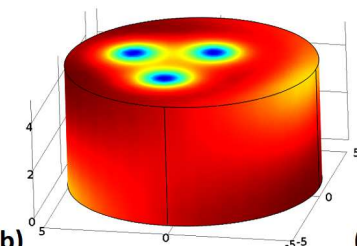

(b)

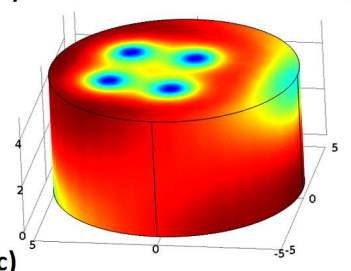

(g)

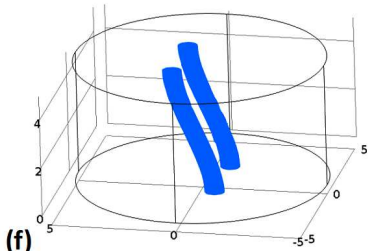

(c)
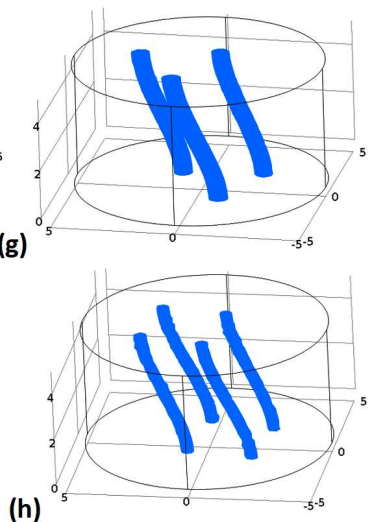

(h)
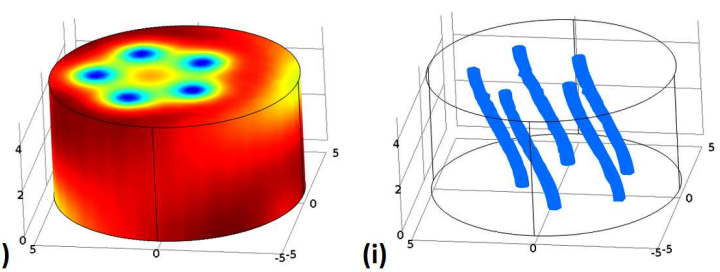

(e)
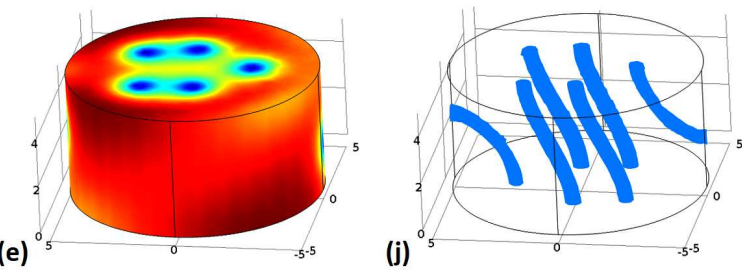

Fig. 4. Vortex tubes in the 3D mesoscopic superconducting cylinder at the field $H / H_{c 2}=0.48$ (a), $H / H_{c 2}=0.56$ (b), $H / H_{c 2}=0.72(\mathrm{c}), H / H_{c 2}=0.76(\mathrm{~d})$, and $H / H_{c 2}=0.82(\mathrm{e})$. The cylinder is in an external uniform magnetic field $H_{\|}\left(\theta=30^{\circ}\right)$. Parts (f) $-(\mathrm{j})$ show the corresponding isosurface plots of vortex tubes of (a)-(e). Blue to red means order parameter range from minimum to maximum.

ant vortex states $[3,25,26]$ with vorticity $L=9-13$ for $H>0.95 H_{c 2}$. It is clear that the $L=0,2-13$ state is a ground state. To investigate the effects of the magnetic field on the multi-vortex states, the selected vortex states and corresponding phases are given in Fig. 3c. One can see that the vortices enter into the cylinder with increasing $H$ value. When encircling a single vortex, the phase of the order parameter changes with $2 \pi$. The strong confinement in the mesoscopic regime prevents the formation of hexagonal structures and we usually obtain ring symmetric structures. With increasing magnetic field vortex enters the sample in the form of tube which was believed to be due to the presence of a surface barrier [29]. 

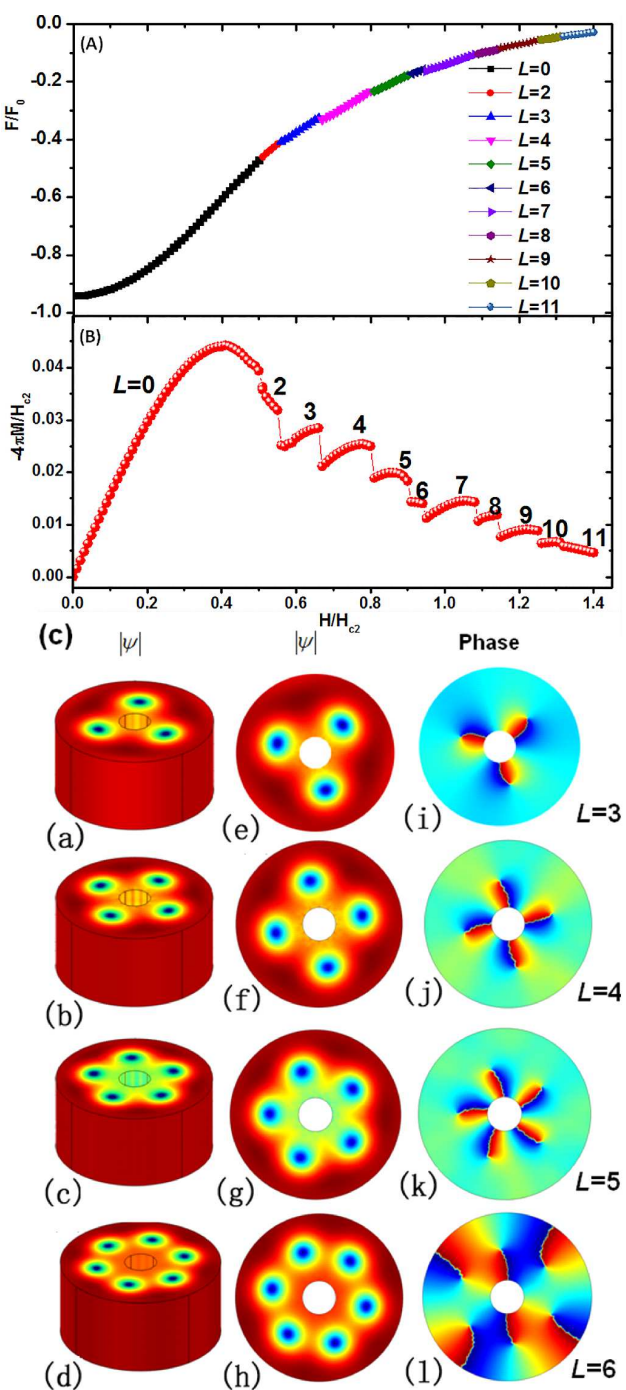

Fig. 5. Free-energy curve (A), magnetization(B) and selected 3D vortex states and corresponding phases (C) in a $3 \mathrm{D}$ mesoscopic superconducting cylindrical shell with the inner radius $R_{\mathrm{i}}=\xi$, outer radius $R_{o}=5 \xi$ and the height $d=5 \xi$. Parts (a)-(d) are the selected $3 \mathrm{D}$ vortex states for $H / H_{c 2}=0.60$ (a), $H / H_{c 2}=0.70$ (b), $H / H_{c 2}=0.86$ (c) and $H / H_{c 2}=0.95$ (d); parts (e)(h) are the corresponding $2 \mathrm{D}$ vortex states (in the $(x, y)$ plane $(z=2.5 \xi))$ as in (a) $-(\mathrm{d})$; parts (i) $-(\mathrm{l})$ are the corresponding phases of the order parameter as in (a) $-(d)$. Blue to red means order parameter range from minimum to maximum, whereas for order parameter phase, it indicates $0-2 \pi$.

Figure 4 shows vortex tubes in the $3 \mathrm{D}$ mesoscopic superconducting cylinder at the tilted fields $H / H_{c 2}=0.48$ (a), $H / H_{c 2}=0.56$ (b), $H / H_{c 2}=0.72$ (c), $H / H_{c 2}=0.76$ (d), and $H / H_{c 2}=0.82(\mathrm{e})$. The cylinder is in an external uniform magnetic field $H_{\|}\left(\theta=30^{\circ}\right)$. Parts $(\mathrm{f})-(\mathrm{j})$ show the corresponding isosurface plots of vortex tubes of (a)(e). Blue to red means order parameter range from minimum to maximum. With a magnetic field not parallel to the $y$ or $z$ axis, the vortices attempt to change their orien- tation accordingly. In mesoscopic superconductors, the screening effects of the Meissner currents that are generated to expel the magnetic field are always maximal in the corners and vortices, as tubes of magnetic flux avoid those regions. Therefore, the end of each vortex tube is bent, and the vortex tube turns gradually to avoid the corner of the sample. At the boundaries of the sample, the supercurrent can only have the component parallel to the surface. Therefore, the vortex endings must be aligned perpendicular to the surface of the sample [30].

Next, we contrast the results of the cylinder to the case of a mesoscopic hollow cylinder. Figure 5 shows the free energy, magnetization and the absolute value of the order parameter and corresponding phases for selected vortex states for a hollow cylinder with the inner radius $R_{\mathrm{i}}=\xi$, outer radius $R_{o}=5 \xi$ and the height $d=5 \xi$. The cylinder is in an external uniform magnetic field $H_{\|}\left(\theta=0^{\circ}\right)$. The energy curve corresponds to the thermodynamic stable pattern, the Meissner phase with no vortices below the first penetration field $H_{p}=0.5 H_{c 2}$ (Fig. 5a). As the applied field is further raised, the vortex tubes enter the sample. Figure $4 \mathrm{~b}$ shows the magnetization as a function of applied magnetic field for the hollow cylinder. We find the multi-vortex states with vorticity $L=2-10$ for $0.50 H_{c 2}<H<1.35 H_{c 2}$, and the giant vortex states with 11 for $H>1.35 H_{c 2}$. To investigate the effects of the magnetic field on the multi-vortex states, the selected vortex states and corresponding phases show the effects of the magnetic field on the multi-vortex states (Fig. 5c). One can see that the vortices enter into the cylinder with increasing $H$ value. Blue to red means order parameter range from minimum to maximum, whereas for order parameter phase, it indicates $0-2 \pi$. The effect of the sample geometry leads to the following differences. First, the Meissner effect becomes more pronounced leading to a large first penetration field $\left(H_{p}=0.5 H_{c 2}\right)$. Second, the jumps in the magnetization curve are much smaller, leading to an almost continuous change in the number of vortex. These results allow us to conclude that the surface barrier for penetration of magnetic field in a hollow cylinder is larger compared to the one in a solid cylinder.

\section{Conclusions}

In this paper we solved the time-dependent GinzburgLandau equations for the three-dimensional mesoscopic superconducting cylinders, and obtained numerical expression in terms of the finite-element method. The effect of the sample geometry leads to the following differences. First, the Meissner effect becomes more pronounced leading to a large first penetration field $H_{p}$. Second, the jumps in the magnetization curve are much smaller, leading to an almost continuous change in the number of vortex. These results allow us to conclude that the surface barrier for penetration of magnetic field in a hollow cylinder is larger compared to the one in a solid cylinder. the larger the inner radius of the superconducting hollow cylinder, the larger the first penetration field $H_{p}$, 
i.e., the magnetic vortices penetrate hardly into the superconductor and the system is hardly magnetized.

\section{Acknowledgments}

his work is sponsored by the National Natural Science Foundationof China (No. 11502141), the Opening Project of Shanghai Key Laboratory of High Temperature Superconductors (14DZ2260700), the Natural Science Foundation of Shanghai (No.17ZR1411400, 15ZR1418700), the Shanghai plateau project (Shanghai University of Electric Power), and the Innovation Program of Shanghai Municipal Education Commission (No. 14YZ132).

\section{References}

[1] G. Carapella, P. Sabatino, G. Costabile, J. Appl. Phys. 112, 083909 (2012).

[2] P. Sabatino, G. Carapella, M. Gombos, J. Appl. Phys. 111, 053912 (2012).

[3] B. Xu, M.V. Milošević, F.M. Peeters, Phys. Rev. B 81, 064501 (2010).

[4] V.A. Schweigert, F.M. Peeters, P.S. Deo, Phys. Rev. Lett. 81, 2783 (1998).

[5] A. Kanda, B.J. Baelus, F.M. Peeters, K. Kadowaki, Y. Ootuka, Phys. Rev. Lett. 93, 257002 (2004).

[6] M.V. Milošević, A. Kanda, S. Hatsumi, F.M. Peeters, Y. Ootuka, Phys. Rev. Lett. 103, 217003 (2009).

[7] T. Cren, L. Serrier-Garcia, F. Debontridder, D. Roditchev, Phys. Rev. Lett. 107, 097202 (2011).

[8] A. Ludu, J. Van Deun, M.V. Milošević, A. Cuyt, F.M. Peeters, J. Math. Phys. 51, 082903 (2010).

[9] Ž.L. Jelić, M.V. Milošević, J. Van de Vondel, A.V. Silhanek, Sci. Rep. 5, 14604 (2015).

[10] A.R. de C. Romaguera, M.M. Doria, F.M. Peeters, Phys. Rev. B 75, 184525 (2007).
[11] M.M. Doria, A.R. de C. Romaguera, F.M. Peeters, Phys. Rev. B 75, 064505 (2007).

[12] G. Carapella, P. Sabatino, C. Barone, S. Pagano, M. Gombos, Sci. Rep. 6, 35694 (2016).

[13] G.Q. Zha, S.P. Zhou, B.H. Zhu, Y.M. Shi, H.W. Zhao, Phys. Rev. B 74, 024527 (2006).

[14] G.R. Berdiyorov, M.V. Milošević, S. Savel'ev, F. Kusmartsev, F.M. Peeters, Phys. Rev. B 90, 134505 (2014).

[15] W.H. Kleiner, L.M. Roth, S.H. Autler, Phys. Rev. 133, A1226 (1964).

[16] G. Carapella, P. Sabatino, G. Costabile, Phys. Rev. B 81, 054503 (2010).

[17] P. Sabatino, G. Carapella, G. Costabile, Supercond. Sci. Technol. 24, 125007 (2011).

[18] G. Carapella, P. Sabatino, M. Gombos, Supercond. Sci. Technol. 30, 025018 (2017).

[19] L.F. Chibotaru, A. Ceulemans, V. Bruyndoncx, V.V. Moshchalkov, Nature (London) 408, 833 (2000).

[20] D.Y. Vodolazov, F.M. Peeters, S.V. Dubonos, A.K. Geim, Phys. Rev. B 67, 054506 (2003).

[21] Y. Enomoto, R. Kato, K. Katsumi, S. Maekawa, Physica C 192, 166 (1992).

[22] S. Adam, F. de Menten de Horne, L. Piraux, S. Michotte, Appl. Phys. Lett. 92, 012516 (2008).

[23] L. Peng, C. Cai, J. Lin, J. Chen, Y. Liu, Y. Zhou, J. Supercond. Nov. Magn. 29, 1197 (2016).

[24] T.S. Alstrøm, M.P. Sřrensen, N.F. Pedersen, S. Madsen, Acta. Appl. Math. 115, 63 (2011).

[25] K.Yu. Arutyunov, D.S. Golubev, A.D. Zaikin, Phys. Rep. 464, 1 (2008).

[26] L. Kramer, R.J. Watts-Tobin, Phys. Rev. Lett. 40 , 1041 (1978)

[27] A. Fortini, E. Paumier, Phys. Rev. B 14, 55 (1976).

[28] L. Peng, C. Cai, J. Low. Temp. Phys. 188, 39 (2017). 